\title{
Miranda
}

Revue pluridisciplinaire du monde anglophone /

Multidisciplinary peer-reviewed journal on the English-

speaking world

19 | 2019

Rethinking Laughter in Contemporary Anglophone Theatre

\section{Xavier Kalck, “We said Objectivist”. Lire les poètes Lorine Niedecker, George Oppen, Carl Rakosi, Charles Reznikoff, Louis Zukofsky.}

\section{Fiona McMahon}

\section{Q OpenEdition}

Édition électronique

URL : http://journals.openedition.org/miranda/22028

DOI : 10.4000/miranda.22028

ISSN : 2108-6559

Éditeur

Université Toulouse - Jean Jaurès

Édition imprimée

Date de publication : 7 octobre 2019

\section{Référence électronique}

Fiona McMahon, "Xavier Kalck,"We said Objectivist”. Lire les poètes Lorine Niedecker, George Oppen, Carl Rakosi, Charles Reznikoff, Louis Zukofsky. », Miranda [En ligne], 19| 2019, mis en ligne le 15 octobre 2019, consulté le 16 février 2021. URL : http://journals.openedition.org/miranda/22028 ; DOI : https:// doi.org/10.4000/miranda.22028

Ce document a été généré automatiquement le 16 février 2021.

\section{cc) (1) $९$}

Miranda is licensed under a Creative Commons Attribution-NonCommercial-NoDerivatives 4.0 International License. 
Xavier Kalck,"We said Objectivist". Lire les poètes Lorine Niedecker, George Oppen, Carl Rakosi, Charles Reznikoff, Louis Zukofsky.

Fiona McMahon

\section{RÉFÉRENCE}

Xavier Kalck, "We said Objectivist”. Lire les poètes Lorine Niedecker, George Oppen, Carl Rakosi, Charles Reznikoff, Louis Zukofsky. Paris : Sorbonne université presses, 2019. 


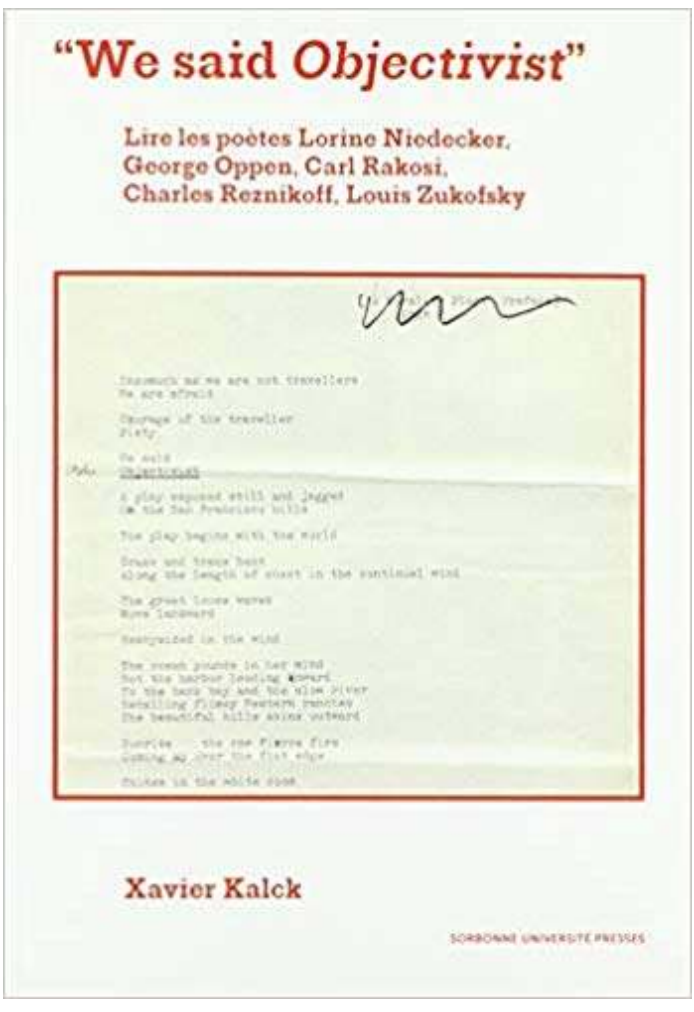

1 La poésie objectiviste n'a pas fini de marquer de son sceau l'actualité littéraire en France. Son lectorat, sans être vaste est hétéroclite, constitué autant d'artistes, de poètes et de traducteurs que de critiques venus des champs anglicistes, américanistes, des lettres comparées, de l'esthétique ou de l'histoire de l'art. En tant que tradition poétique, son jaillissement sur la création actuelle a été salué encore récemment par les poètes Philippe Blanchon, Éric Giraud et le critique d'art François Coadou ${ }^{1}$. S'il fallait encore des preuves de la centralité de l'exemple objectiviste, il suffirait d'observer la production encore plus récente de traductions inédites des poètes objectivistes (en 2018, poèmes de Carl Rakosi et de Charles Reznikoff chez les Éditions unes, Éditions Nous ou Éditions La Barque).

2 Si aujourd'hui l'intérêt pour la critique française à l'égard de ces poètes n'est plus à démontrer, l'ensemble des œuvres poétiques que l'historiographie littéraire réunit sous l'étiquette d'« objectiviste » a traversé le vingtième siècle sans grand fracas, les poètes américains George Oppen, Carl Rakosi, Charles Reznikoff et Louis Zukofsky ayant tardé à trouver leur place à l'intérieur du canon poétique américain. Plus radicalement obscures encore sont les quelques figures rattachées à cette filiation par la critique à la fin des années quatre-vingt-dix : la poétesse américaine Lorine Niedecker et l'anglais Basil Bunting.

3 Malgré la relative discrétion de ces œuvres au sein des universités françaises, l'ouvrage de Xavier Kalck, “We said Objectivist”. Lire les poètes Lorine Niedecker, George Oppen, Carl Rakosi, Charles Reznikoff, Louis Zukofsky (Sorbonne université presses, 2019) ne manquera pas de trouver ses lecteurs, férus de poésie américaine, mais aussi ceux, nombreux chez les poètes français contemporains, qui sont sensibles aux traditions poétiques américaines et parfois traducteurs de ces mêmes poètes objectivistes (J. Roubaud, Y. di Manno, J-P Auxeméry, A-M Albiach, entre autres). Kalck recense des traducteurs français des œuvres objectivistes à cette date, proposant une liste exhaustive dans 
l'introduction de son ouvrage ${ }^{2}$. La traversée remarquablement minutieuse du paysage critique que l'on y découvre rappelle l'imbrication constante de l'écriture objectiviste dans les principales étapes de la poésie américaine du $20^{e}$ siècle. Kalck retrace cette histoire et celle de la critique américaine, à commencer par les études fondatrices initiant le public et le monde universitaire américains à ces œuvres (l'ouvrage clé de Michael Heller paru en 1985, A Conviction's Net of Branches : Essays on the Objectivist Poets and Poetry ou les entretiens menés par l'universitaire américain L.S. Dembo en 1972). Il rend hommage également à l'inestimable contribution de Serge Fauchereau (Lecture de la poésie américaine, 1968), auteur d'une étude en langue française qui situe les objectivistes dans le paysage poétique américain $\mathrm{du} 20^{\mathrm{e}}$ siècle et qui introduit le lectorat français de manière plus large, comme le rappelle Yves di Manno, à une « étrange galaxie poétique ${ }^{3}$ ".

4 L'ambition de l'ouvrage présent, fruit d'une thèse de doctorat soutenue en 2007 est précisément d'éclairer la place des objectivistes dans cet univers poétique et d'appréhender chemin faisant, à l'aide de micro-lectures, la singularité prosodique de chaque poète sans négliger les circonstances géopolitiques présidant à la composition. La démarche de Kalck enrichit de manière didactique et pertinente la tradition critique monographique en proposant un chapitre distinct pour explorer les caractéristiques individuelles formant la poétique de chaque œuvre. Doté d'une belle érudition, Kalck produit un texte animé par une plume directe et assurée.

5 L'ouvrage se divise en cinq chapitres où sont proposés selon une répartition éditoriale parfaitement équilibrée (une quarantaine de pages à chaque fois) des outils d'analyse des poétiques de Niedecker, Oppen, Rakosi, Reznikoff et Zukofsky. L'ensemble est encadré par une introduction dans laquelle Kalck inventorie les principales études consacrées aux États-Unis et en France aux poètes objectivistes, avant d'enchaîner sur un volet biographique succinct et utile composé de "cinq portraits». L'ouvrage se termine par une conclusion intitulée, "Ruines du particulier », qui met en regard ce que Zukofsky nommera "les faits historiques et contemporains particuliers ${ }^{4}$ » et les enjeux d'une poésie du banal ou du quotidien, si souvent assimilée aux œuvres objectivistes.

6 L'ordonnancement de l'ouvrage est à l'image de la clarté critique dont fait preuve l'étude de Kalck. Celle-ci s'ouvre sur la réception de la poésie objectiviste, longtemps balisée par des efforts d'y trouver un projet commun ou d'y consacrer une " tradition ", comme s'est employé à le faire l'un de ses premiers lecteurs critiques, l'américain Charles Altieri en 1979. Selon Kalck, ce qui constitue l'un des points d'achoppement dans ce paysage critique est le fait que "The Objectivist Tradition " peine à rendre compte des fortunes diverses de ses différents acteurs à travers une période longue, des années 30 jusqu'aux années 70 . En effet, certaines des premières lectures critiques, dont celui d'Altieri, rencontre chez Kalck une force argumentative qui s'attarde sur la nécessité d'affiner des positions théoriques. Remettre à plat ainsi l'historiographique est une stratégie critique efficace, mais le lecteur pourra être frappé par le caractère récurrent de la méthode employée ici qui consiste en une opposition frontale aux propos des auteurs cités, parmi lesquels figurent les poètes qui les premiers ont sensibilisé le lectorat à la poétique des objectivistes (Emmanuel Hocquard en France ou Michael Heller et plus récemment Rachel Blau Duplessis aux États-Unis).

7 Reste que le regard interrogateur que l'auteur pose sur l'objet de ses recherches est fondé sur une grande connaissance des poétiques américaines $\mathrm{du} 20^{\mathrm{e}}$ siècle. Son étude 
est tournée ainsi vers l'ensemble des œuvres objectivistes américaines mais se garde d'offrir un quelconque discours général dans lequel viendraient se télescoper les spécificités de chaque œuvre. De fait, en s'emparant de la question de l'héritage objectiviste, cet ouvrage se donne pour ambition de mettre à nu des lectures critiques du projet objectiviste. Kalck attend de l'activité critique qu'elle dépasse un certain nombre d'écueils méthodologiques, en s'émancipant des «formules vagues» (13) longtemps arrimées aux termes du programme fondateur énoncés par Louis Zukofsky en 1931 - c'est toutefois une entreprise à laquelle s'attelle la critique de manière régulière - en se délestant d'une partialité théorique trop présente et en demeurant vigilante face à la question de la « cohérence du projet objectiviste » (13).

C'est par la méditation attentive sur l'œuvre de Charles Reznikoff et sa réception que Kalck choisit d'inaugurer son étude. Les motifs prosodiques et philosophiques de son écriture, singuliers par leur mesure et leur humilité produiront des échos chez Zukofsky, Oppen, Rakosi et Niedecker. Kalck célèbre les seuils langagiers exhibés par les poèmes de Reznikoff, les difficultés qu'ils posent pour la réception d'une œuvre qui semble refuser le défi de la transcendance pour se réfugier dans une vision de concrétude objective. Les outils d'analyse de Kalck permettent de montrer les limites d'une telle lecture au profit d'une position dialectique. Celle-ci consiste à envisager l'exemplarité du poète du point de vue de l'articulation sous-jacente qui fait se rencontrer le formalisme associé au modernisme poétique et l'héritage culturel juif de Reznikoff. À ce titre, ce premier chapitre comporte une analyse vigoureuse et éclairante de l'analogie à laquelle a recours une certaine critique américaine entre la "textualité juive traditionnelle» (en citant notamment Norman Finkelstein, l'un des critiques les plus productifs de la question de la sécularisation de la culture juive) et «l'expérimentation poétique d'avant-garde » (68). Après Stephen Fredman, Stephen Paul Miller ou Daniel Morris ${ }^{5}$, Kalck rejoint ainsi un débat de longue date sur l'appropriation en poésie américaine d'un régime liturgique ou d'une démarche midrashique et de manière générale, sur la place du judaïsme dans la littérature américaine.

9 Le deuxième chapitre, qui s'ouvre par le titre évocateur «La disparition de Zukofsky », se lit comme un plaidoyer pour un retour au «travail du texte» (94) face à la récupération du poète à la fois comme "figure tutélaire du modernisme poétique américain » (90) et « caution du postmodernisme triomphant » (91). Selon Kalck, avant d'envisager de nouveaux modèles interprétatifs de "A", l'œuvre majeure de Zukofsky, il s'agit de mettre à distance les modalités pratiques et théoriques de la critique poundienne tout comme des positionnements théoriques des «Language Poets » dans les années 80 et 90 . Pour cerner la spécificité prosodique du projet de Zukofsky, Kalck s'appuie notamment sur une étude de Cid Corman, proposant en conclusion de ce chapitre un retour incisif vers l'anthologie que Zukofsky consacre à l'art poétique, $A$ Test of Poetry (1948).

10 Pour le lectorat français, le troisième chapitre, intitulé «Les échelles de Carl Rakosi » est essentiel, en ce qu'il contribue aux efforts trop rares pour rendre compte de l'œuvre de ce poète. Kalck revient sur une nouvelle "disparition » dans le paysage poétique américain, puisque si Rakosi est présent dans An "Objectivists" Anthology, (1932) ${ }^{6}$ aux côtés de Zukofsky, Oppen et Reznikoff, il ne fera paraître de nouveaux poèmes qu'en 1967, avec le volume Amulet (aujourd'hui traduit en français par J-P Auxeméry). L'analyse proposée ici fait ressortir autant la «dimension méditative» chez Rakosi 
(173) que ce que Kalck nomme la "minoration » (155) du poème aux prises avec les « capacités expressives et représentatives » (154) du langage.

11 Le chapitre suivant consacré à Lorine Niedecker participe de la démarche à l'œuvre dans l'ensemble de l'ouvrage, celle qui consiste à contester les méthodologies critiques utilisées pour l'analyse des œuvres objectivistes. "Lorine Niedecker: ancrage et abstraction" s'efforce de prendre à rebours l'image d'une œuvre marquée par la simplicité, du fait de son dépouillement et de l'anonymat associé à la trajectoire biographique du poète. Dans le dernier chapitre de son ouvrage, Kalck, qui est déjà l'auteur d'une étude parue chez Peter Lang en 2017 (George Oppen's Poetics of the Commonplace), propose une analyse synthétique de la poésie d'Oppen à travers la lecture minutieuse d'un seul poème, "The Lighthouses ", composé en 1975 et adressé par Oppen à Zukofsky. Ici, à nouveau, Kalck souligne son parti pris : il s'emploie à tenir les dynamiques internes de la composition à distance d'approches théoriques jugées inadaptées, pour mettre au jour les mécanismes de la prosodie.

Dans sa conclusion, Kalck ramène l'héritage des objectivistes au contexte contemporain et s'attaque à la problématisation de l'objet et du banal dans la poésie américaine en esquissant un détour par le cinéma américain récent. L'appropriation du motif du prosaïque par Jim Jarmusch dans son film Paterson (2016) est certes caricaturale et Kalck n'a aucune peine à nous le démontrer. De fait, il réussit son pari qui consiste à nous faire nous attarder dans ce parcours du paysage objectiviste non pas sur «l'authenticité du banal » (267) mais sur «L'étrangeté qui perturbe la coïncidence du particulier et du familier » (271). Contempler avec Kalck cette " étrangeté », la distance qui sépare le regard objectiviste de la tradition poétique américaine ou de l'américanité donne également à penser la subtile déchirure de l'expérience qu'ont traversée individuellement ces poètes. Cette invitation est l'un des nombreux points forts qui font de cette étude une contribution importante au champ de recherche faisant avancer la connaissance en France des poétiques américaines.

\section{NOTES}

1. Des objectivistes au Black Mountain College. École supérieure d'art de Toulon Provence Méditerranée : La Nerthe, 2014.

2. Nous nous permettons de rappeler la notice bibliographique suivante: Charles Reznikoff, D’abord il y a la nécessité, trad. Jules Henri Julien. L'ours blanc. Genève, Héros-Limite, 2014, 2-16.

3. Objets d'Amérique. Série américaine. Paris : Josi Corti, 2009, 14.

4. Louis Zukofsky, Prepositions +: The Collected Critical Essays. Middleton (Conn.), Wesleyan UP, 2000, 12. Cité par X. Kalck, 275.

5. A Menorah fro Athena: Chalres Reznikoff and the Jewish Dilemmas of Objectivist Poetry, 2001 ; Stephen Paul Miller et Daniel Morris (dir.), Radical Poetics and Secular Jewish Culture, Tuscaloosa, University of Alabama Press, 2010.

6. Ed. Louis Zukofsky, Le Beausset, Var, France; New York, PO Box 3 Station F: To, Publishers, 1932. 
INDEX

Mots-clés : poésie américaine, objectivistes, tradition critique, prosodie

Keywords : American poetry, objectivists, critical tradition, prosody

\section{AUTEURS}

\section{FIONA MCMAHON}

Maître de conférences HDR

Université de Bourgogne

Fiona.Mc-Mahon@u-bourgogne.fr 